ORIGINAL ARTICLE

\title{
Discordant quantitative detection of putative biomarkers in nodal micrometastases of colorectal cancer: biological and clinical implications
}

\author{
S L Kong, M Salto-Tellez, A P K Leong, Y H Chan, E S C Koay
}

J Clin Pathol 2005;58:839-844. doi: 10.1136/icp.2004.023853

See end of article for authors' affiliations

.....................

Correspondence to:

Dr E S C Koay, Department

of Pathology, National

University of Singapore,

10 Kent Ridge Crescent,

Singapore 119260;

patkoaye@nus.edu.sg

Accepted for publication 4 January 2005

\begin{abstract}
Aims: Nodal expression of the carcinoembryonic antigen (CEA), cytokeratin 20 (CK20), and guanylyl cyclase $C$ (GCC) genes was measured in tandem in patients with colorectal cancer (CRC) to assess whether there would be sufficient agreement between these markers in their ability to detect micrometastasis to qualify one of them as a universal marker, and whether frozen and paraffin wax embedded tissues would yield similar results.

Methods: One hundred and seventy five frozen lymph nodes (FT) and 158 formalin fixed, paraffin wax embedded lymph nodes (PET) from 28 CRC cases were analysed using gene specific quantitative real time polymerase chain reaction, carried out on the LightCycler ${ }^{\circledR}$ system with SYBR Green chemistry.

Results: There was significant disparity in positive detection of the three biomarkers in FT versus PET, with notable agreement achieved only for CEA (66.6\%) in FT versus PET in Dukes' B disease, and between CK2O and GCC (44.6\%) in FT, also in Dukes' B disease. One patient with full concordance in all three tumour markers with both tissue types suffered a relapse and died within two years of follow up.

Conclusions: There was considerable discordance in the positive detection of the three tumour markers in both tissue types (FT versus PET). This brings into question whether using a single tumour marker to detect micrometastasis in one tissue type (FT or PET) is adequately representative, and challenges the concept of universal markers for molecular CRC metastatic detection. Multiple tumour markers would predict more accurately the metastatic potential of Dukes' B CRCs.
\end{abstract}

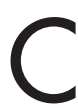
olorectal cancer (CRC) is the third leading cause of cancer and cancer related mortality worldwide, with a strong association between lymph node (LN) metastases and poor outcome. However, a large proportion (30$40 \%$ ) of patients with histologically negative tumours often suffer recurrence after surgery, presumably as a result of undetected, disseminated micrometastases at the time of diagnosis. ${ }^{1-3}$ Because surgery and adjuvant chemotherapy are the standard of care for Dukes' C CRC but not for Dukes' B disease, better assessment of prognosis in patients with Dukes' B CRC could allow the selective use of adjuvant treatment in some and prevent unnecessary treatment in others. ${ }^{4}$ In this context, immunohistochemistry has shown a lack of reproducibility and an inability to provide quantitative data. ${ }^{5}$ Even if serial sectioning and immunohistochemical staining can detect occult micrometastases in up to $25 \%$ of node negative patients, this would be too cumbersome and costly to be practised on a routine basis. ${ }^{6}$ This limitation has resulted in the extensive evaluation of reverse transcriptasepolymerase chain reaction (RT-PCR) based assays as routine clinical tools in diagnostic pathology. However, we have shown how the intrinsic variability in the applied methodology has prevented meaningful comparison of the results from different laboratories. ${ }^{7}$ Arguably, the use of quantitative real time PCR (R-PCR) may help to resolve these problems by providing a more sensitive, reliable, and measurable detection of disseminated cancer cells in CRC, because R-PCR has several advantages over other traditional PCR strategies. ${ }^{8-10}$ In the R-PCR system, mRNA expression is quantified during the logarithmic phase of PCR amplification, which provides a more reliable method of gene quantification. In addition, the fluorescence detection system of R-PCR is more sensitive than conventional ethidium bromide staining and no postPCR procedure is required.

\begin{abstract}
"Better assessment of prognosis in patients with Dukes' B colorectal cancer could allow the selective use of adjuvant treatment in some and prevent unnecessary treatment in others"
\end{abstract}

We developed an R-PCR assay on the LightCycler ${ }^{\circledR}$ system (Roche Molecular Systems, Indianapolis, Indiana, USA), one of several highly automated, rapid, and high throughput R-PCR systems, to detect the expression of mRNA encoding carcinoembryonic antigen (CEA), cytokeratin 20 (CK20), and guanylyl cyclase C (GCC) as a surrogate for occult metastases of CRC affected lymph nodes. CEA, a well established tumour marker, is expressed in most gastrointestinal cancers and has been widely used to monitor and detect early relapse in patients with CRC. ${ }^{311}$ CK20 is highly expressed in gastric and intestinal epithelium and appears to be relatively specific for gastrointestinal tract adenocarcinoma. ${ }^{12}$ GCC, a receptor that mediates fluid and electrolyte secretion, ${ }^{13}$ is only expressed in mucosal cells lining the intestine, from the duodenum to the rectum, but is absent in other extraintestinal tissue. As such, GCC may be a unique biomarker for metastatic CRC. ${ }^{14}$ We tested both formalin fixed, paraffin wax embedded material and frozen material obtained from the same LN of patients with CRC. We distinguished baseline constitutional expression from cancer specific expression of these markers by generating a cutoff value for each marker in each type of tissue, and studied the effects of applying the results

Abbreviations: CEA, carcinoembryonic antigen; CK20, cytokeratin 20; $\mathrm{CRC}$, colorectal cancer; $\mathrm{Ct}$, crossing point; $\mathrm{FT}$, frozen tissue; GCC, guanylyl cyclase C; LN, lymph node; PBMN, peripheral blood mononuclear; PCR, polymerase chain reaction; PET, paraffin wax embedded tissue; R-PCR, quantitative real time polymerase chain reaction; $R T$, reverse transcriptase 
generated from these R-PCR based quantitative assays to the potential molecular re-staging of Dukes' B CRC.

\section{MATERIALS AND METHODS}

\section{Cell lines}

We purchased the DLD-1 cell line, derived from human colorectal adenocarcinoma with epithelial morphology, from the American Type Culture Collection (ATCC, Manassas, Virginia, USA).

\section{Patients and tissues}

The experimental design is similar to the one reported previously. ${ }^{7}$ Briefly, we studied a total of 220 LNs from 28 consecutive patients with CRC (two Dukes' A, 11 Dukes' B, 15 Dukes' C) from our diagnostic routine workload. The nodes were sampled in the fresh state immediately after surgery. Half of each resected $\mathrm{LN}$ was snap frozen at $-152^{\circ} \mathrm{C}$, and the other half was fixed for 24 hours in $10 \%$ formalin and embedded in paraffin wax (each LN individually). There were 35 extra frozen LNs without corresponding paraffin wax embedded material from 13 patients because the paraffin wax embedded tissue (PET) had been fully used for diagnostic purposes before the molecular study was carried out. In six other patients, there was insufficient frozen tissue (FT) for successful PCR amplification, resulting in an extra 18 paraffin wax embedded nodes being available for study, with no frozen equivalents. An important aspect of our study design was the inclusion of appropriate controls. We incorporated both frozen and paraffin wax embedded

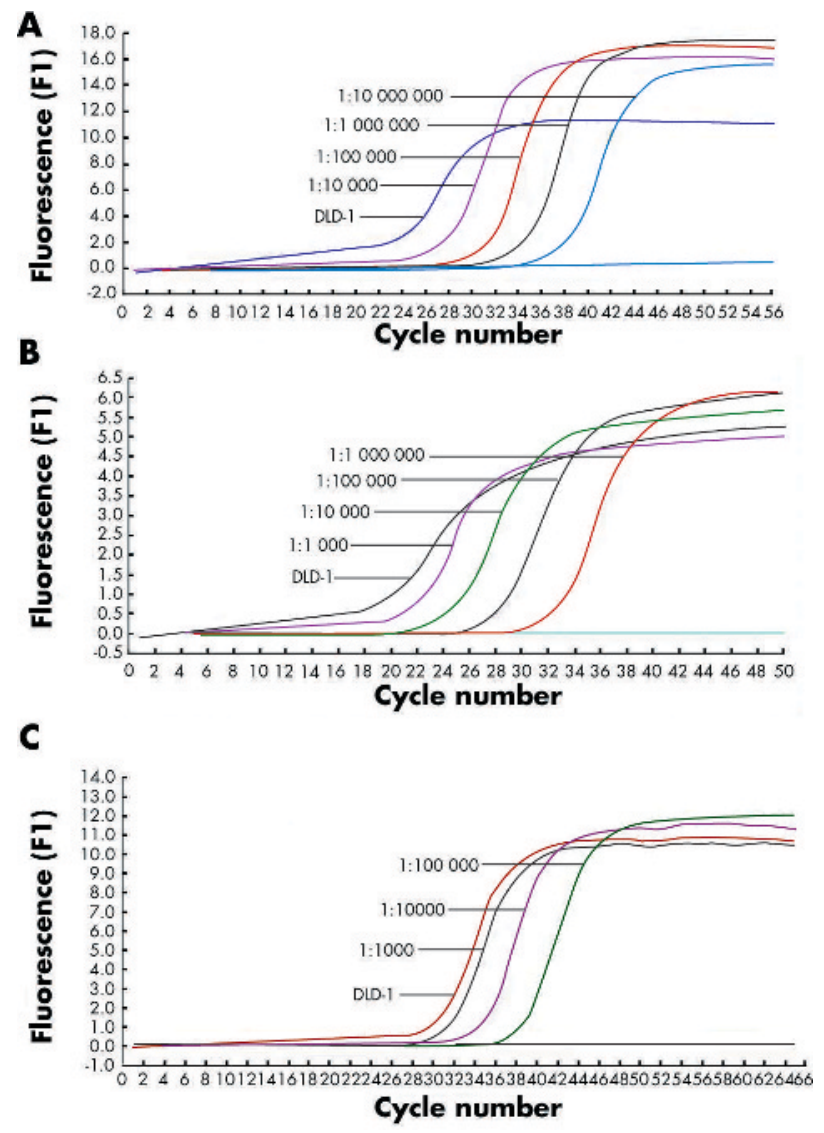

Figure 1 Sensitivity of the quantitative real time polymerase chain reaction assay. Amplification runs for (A) CEA, (B) CK20, and (C) GCC were performed with serial dilutions of DLD-1 cells spiked into known concentrations of peripheral mononuclear cells obtained from a healthy donor. Reactions with dilutions greater than the highest dilution depicted in the plots and negative controls yielded no amplified products.
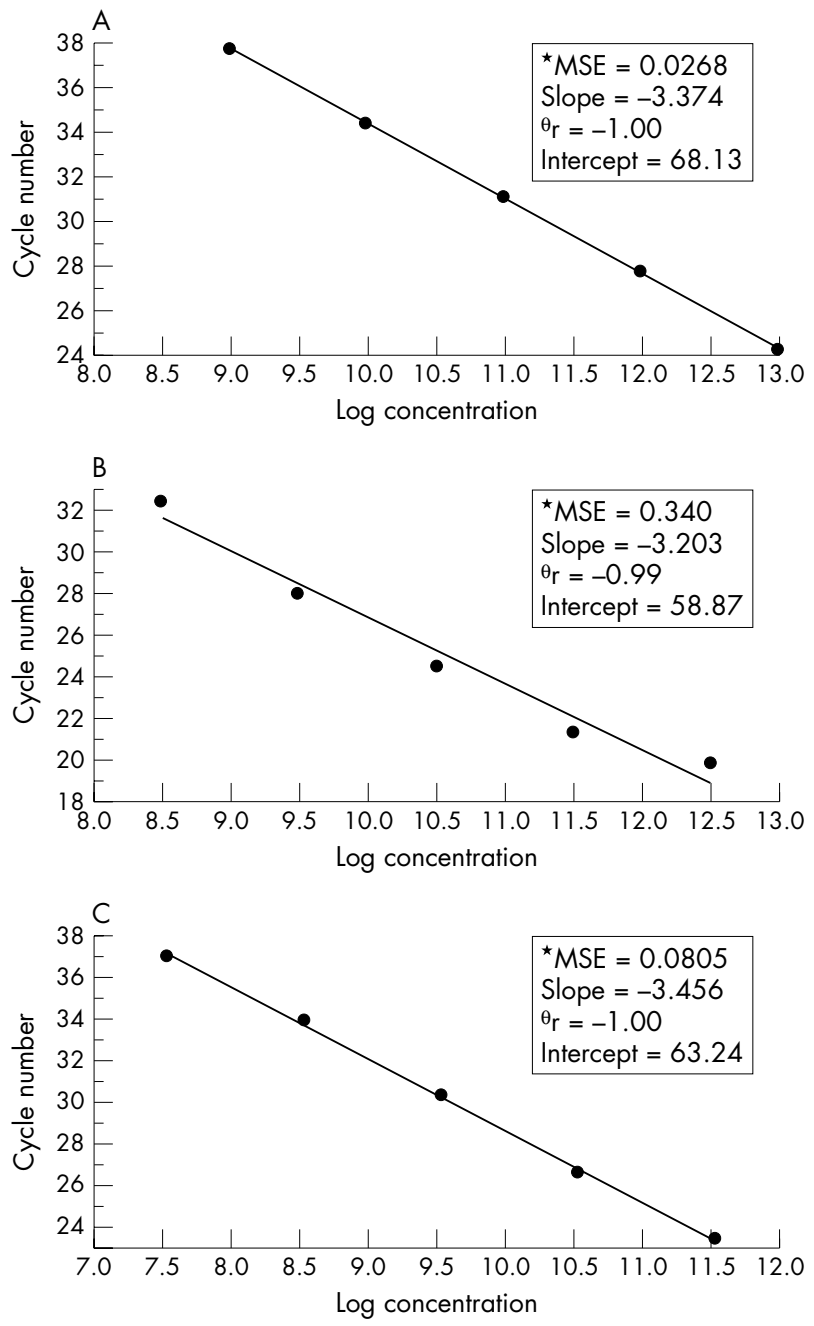

Figure 2 Standard curves for CEA, CK20, and GCC mRNA estimation, constructed by plotting the crossing point cycle numbers against the log concentrations of DLD-1 cells. The expression of (A) CEA, (B) CK20, and (C) GCC mRNA for patient samples was calculated with reference to the appropriate curve. MSE, mean square error; ${ }^{\theta} r$, regression.

material from nine LNs taken from patients diagnosed with diverticulitis and no history of cancer, to serve as negative control samples. We also included the histologically positive LNs from patients with Dukes' C CRC (again, both FT and PET nodal tissues from these patients) and the DLD- 1 cell line as positive controls.

\section{RNA extraction}

The Paraffin Block RNA isolation kit (Ambion Inc, Austin, Texas, USA) was used to extract RNA from paraffin wax embedded LNs. Total RNA was extracted from frozen LNs with Tri Reagent ${ }^{\circledR}$ (Molecular Research Center Inc, Cincinnati, Ohio, USA), according to the manufacturer's recommended protocol. DNase I treatment was incorporated to eliminate genomic DNA contamination of the RNA extract. Total RNA from the DLD-1 CRC cell line was isolated in a similar manner to serve as a positive control for the cDNA synthesis and R-PCR steps.

\section{RT-PCR}

RT-PCR was performed using the Advantage ${ }^{\text {TM }}$ RT-PCR kit (Clontech Laboratories, Palo Alto, California, USA). The optimal RT-PCR was carried out in a reaction consisting of $8 \mathrm{U}$ of Moloney murine leukaemia virus reverse transcriptase, 
Moloney murine leukaemia virus buffer, $4 \mathrm{pmol} / \mu \mathrm{l}$ of random hexamers, $0.5 \mathrm{mmol} /$ litre of dNTPs, $10 \mathrm{nmol} /$ litre of dithiothreitol, $1.2 \mathrm{U} / \mu \mathrm{l}$ of RNase inhibitor, and $1 \mu \mathrm{g}$ of total RNA, made up to $25 \mu \mathrm{l}$ with diethyl pyrocarbonate treated water. Reaction tubes were incubated at $70^{\circ} \mathrm{C}$ for five minutes, $42^{\circ} \mathrm{C}$ for 60 minutes, and $95^{\circ} \mathrm{C}$ for 5 minutes.

A separate RT-PCR run for the nodal expression of the housekeeping abl gene,,$^{15}$ which serves as an internal control for the RNA extraction and cDNA synthesis steps, was carried out.

\section{Quantitative R-PCR}

R-PCR was carried out using SYBR Green I chemistry and the LightCycler Instrument. We used SYBR Green I chemistry rather than hybridisation probes to gain the additional flexibility for parallel (simultaneous) validation of several genes.

R-PCR amplification was carried out in $10 \mu \mathrm{l}$ of reaction mixture consisting of $0.5 \mu \mathrm{mol} /$ litre of primer pairs, as described in our previous study. ${ }^{76-18}$ Template cDNA ( $\left.1 \mu \mathrm{l}\right)$ was added to the reaction mixture (LightCycler FastStart DNA Master SYBR Green I; Roche Diagnostics GmbH, Mannheim, Germany), which contained FastStart Taq DNA polymerase, reaction buffer, dNTP mix (with dUTP instead of dTTP), SYBR Green I dye, FastStart enzyme, and $3 \mathrm{mmol} /$ litre $\mathrm{MgCl}_{2}$ for the CEA and GCC reaction or $4 \mathrm{mmol} /$ litre $\mathrm{MgCl}_{2}$ for the CK20 reaction.

The emitted fluorescence was captured and analysed using the LightCycler analysis software version 3.5. The crossing points $(\mathrm{Ct})$, the beginning of the exponential phase during amplification cycles for each reaction, were determined by the second derivative maximum algorithm and arithmetic baseline adjustment.

The PCR amplified products were differentiated by analysing the melting curves, the shapes of which are a function of the GC content, length, and sequence of the amplified gene fragment. During the initial optimisation of our R-PCR assays, PCR amplified products were subjected to gel electrophoresis and the amplicon identity was confirmed by sequence analysis on the ABI PRISM ${ }^{\mathrm{TM}} 377$ DNA sequencer (Applied Biosystems, Foster City, California, USA).

A standard curve was prepared from 10-fold serial dilutions of cDNA prepared from the CEA, CK20, and GCC mRNA expressing DLD-1 cancer cells, by plotting the relation between the $\mathrm{Ct}$ and the logarithm of copy numbers of amplified products. Five standards (serial dilutions) and a negative control without template were included in each run. The copy numbers of each sample for each nanogram of RNA were extrapolated from the linear standard curve and the $\mathrm{Ct}$ values of each sample.

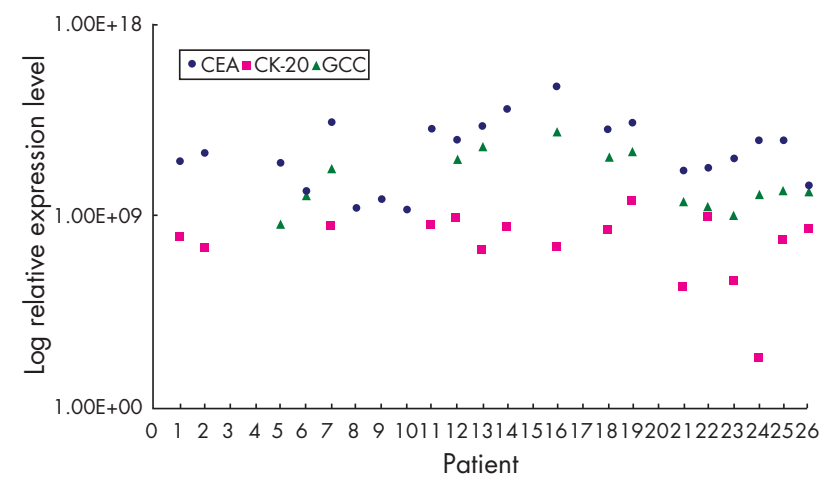

Figure 3 The relative expression of CEA, CK20, and GCC in frozen lymph nodes from patients with Dukes' $B$ and $C$ colorectal cancer.

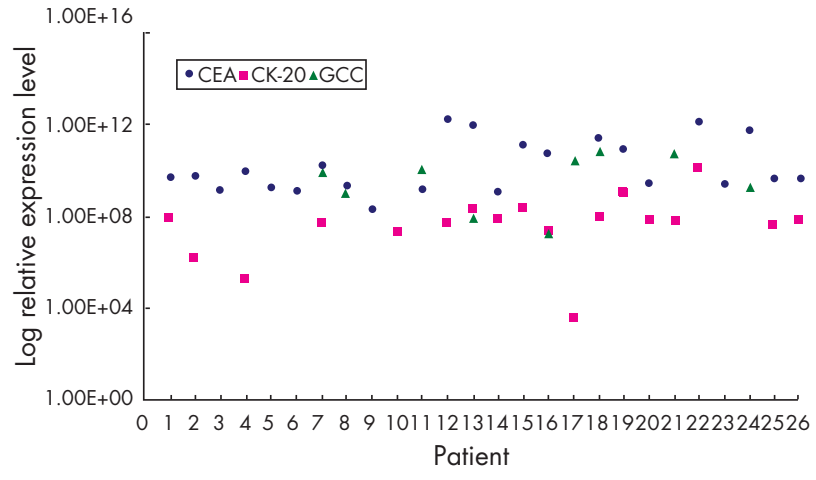

Figure 4 The relative expression of CEA, CK20, and GCC in fixed lymph nodes from patients with Dukes' B and C colorectal cancer.

To determine the sensitivity of our CEA, CK20, and GCC specific R-PCR, cell dilutions were prepared by diluting DLD-1 cells with normal peripheral blood mononuclear (PBMN) cells from a healthy donor as follows: 1/1000, 1/10 000, 1/ $100000,1 / 1000000$, and 1/10 000000 . Capillary reactions containing only DLD-1 cells or PBMN cells were included in the run to serve as positive or negative controls. Extra positive and negative controls included histologically positive lymph nodes from patients with Dukes' $\mathrm{C}$ disease and negative lymph nodes from patients with no malignancy (diverticulitis), respectively.

\section{Statistical analysis}

Fisher's exact test and the binomial test were performed to measure the correlation between the different tumour markers and tissue types. All analyses were performed using the SPSS 11.0 program, with significance set at $\mathrm{p}<0.05$.

\section{RESULTS}

Agarose gel electrophoresis, sequencing, and LightCycler melting curve analysis of the amplified products showed the correct sizes, sequences, and melting patterns for the three markers. Carryover contamination was minimised because no post-amplification procedure is needed. The sensitivity of the real time fluorescence PCR assays is: one copy of a CEA expressing cell in a background of $10^{7}$ copies of PBMN cells; one copy of a CK20 expressing cell in a background of $10^{6}$ copies of PBMN cells; and one copy of a GCC expressing cell in a background of $10^{5}$ copies of PBMN cells (fig 1). The CEA, CK20, and GCC mRNA values were

Table 1 Discordance in positive rates for different tumour markers examined in different tissue types for Dukes' B and C colorectal cancers

\begin{tabular}{lllll}
\hline & \multicolumn{2}{l}{$\begin{array}{l}\text { Discordance } \\
\text { for Dukes' B } \\
\text { cases }\end{array}$} & \multicolumn{3}{l}{$\begin{array}{l}\text { Discordance } \\
\text { for Dukes' C Value }\end{array}$} \\
& cases & p Value \\
\hline CEA (PET) v CK20 (PET) & $27.3 \%$ & 0.015 & $40.0 \%$ & 0.000 \\
CEA (PET) v GCC (PET) & $45.5 \%$ & 0.000 & $46.7 \%$ & 0.000 \\
CK20 (PET) v GCC (PET) & $36.4 \%$ & 0.000 & $60.0 \%$ & 0.000 \\
CEA (FT) v CK20 (FT) & $22.2 \%$ & 0.071 & $23.1 \%$ & 0.025 \\
CEA (FT) v GCC (FT) & $33.3 \%$ & 0.000 & $15.4 \%$ & 0.135 \\
CK20 (FT) v GCC (FT) & $55.6 \%$ & 0.001 & $38.5 \%$ & 0.000 \\
CEA (PET) v CEA (FT) & $33.3 \%$ & 0.008 & $7.7 \%$ & 0.487 \\
CK20 (PET) v CK20 (FT) & $33.3 \%$ & 0.008 & $23.1 \%$ & 0.025 \\
GCC (PET) v GCC (FT) & $44.4 \%$ & 0.001 & $38.5 \%$ & 0.000 \\
\hline
\end{tabular}

The $p$ values are based on the binomial test at $95 \%$ comparison with significance set at $\mathrm{p}<0.05$.

FT, frozen tissue; PET, paraffin wax embedded tissue. 
calculated with reference to the respective standard curve for each marker, in which the slope for linear regression was consistently less than 4.0, indicating high PCR amplification efficiency $(>89 \%$ ) (fig 2 ). Table 1 provides a summary of patient data analysed by CEA, CK20, and GCC specific R-PCR and the relative degree of expression of the positive samples is tabulated in figs 3 and 4 for frozen and fixed tissues, respectively.

At the outset, a total of 28 CRC cases from 28 patients (two Dukes' A, 11 Dukes' B, and 15 Dukes' C) were recruited for our study. Fifty eight (48 PET, 10 FT) nodes in which the abl gene (included as an internal control) was not amplified were subsequently excluded from the database to eliminate false negative results. The experimental data obtained from the remaining 164 frozen LNs (94.3\%) and 116 fixed LNs (70.7\%) from these 28 cases, which confirmed that both RNA extraction and R-PCR amplification were successfully carried out, were retained in the database for statistical analysis.

None of the LNs from patients with Dukes' A disease showed amplification of the GCC fragment. For CEA and CK20, the maximum expression values seen in Dukes' A LNs (minimally invasive carcinomas with no obvious LN metastases) for both frozen and fixed tissues were used as cutoff values for the appropriate tissue type, and any values above the highest Dukes' A LN value were considered positive, to distinguish between baseline constitutional expression and cancer specific expression, thus minimising false positive results. Table 2 shows the results of Dukes' B and C cases with gene expression values above the cutoff values.

CEA mRNA was detected in the frozen LNs of six of nine patients with Dukes' B and in all patients with histologically positive Dukes' C disease. For the fixed LNs (PET), four of 11 patients with Dukes' B and 14 of 15 with Dukes' C disease showed positive amplification of CEA amplicons, indicating a high sensitivity for our assay. CK20 was detected in the frozen LNs of four of nine patients with Dukes' B and 10 of 13 with Dukes' $\mathrm{C}$ disease, and in the fixed LNs of three of $\mathrm{Il}$ patients with Dukes' B and 10 of 15 with Dukes' C disease. GCC mRNA was detected in the frozen LNs of three of nine patients with Dukes' B and 11 of 13 with Dukes' C. In comparison, the fixed LNs of three of 11 patients with Dukes' $\mathrm{B}$ and seven of 15 with Dukes' C disease showed positive amplification of GCC amplicons.

Our results indicate that seven of 11 patients with morphological Dukes' B disease showed the presence of CEA mRNA transcripts in their LNs (that is, were R-PCR positive), either in FT or PET, whereas five of the 11 patients with Dukes' B disease were GCC or CK20 R-PCR positive either in FT or PET, or both. Of the 11 patients with Dukes' B disease, one patient (number 7) who was positive for all tumour markers with both tissue types suffered a relapse and died within two years of follow up.

Statistical analysis showed that strong associations between the Dukes' classification and our R-PCR results were seen only for CEA in PET and FT (Fisher's exact test, $\mathrm{p}=0.001$ and $\mathrm{p}=0.002$, respectively) and GCC in FT $(p=0.012)$. There were either no significant associations, or only weak associations, between the Dukes' classification and the results for CK20 both in PET and FT (Fisher's exact test, $p=0.076$ and $p=0.114$, respectively) and for GCC in PET $(\mathrm{p}=0.395)$. The binomial test showed that there was significant discordance between the positive rates for the different tumour markers examined in the different tissue types (table 1). The only significant correlation between FT and PET was for the CEA marker in patients with Dukes' C disease.

\section{DISCUSSION}

The detection of micrometastases in regional LNs by a molecular method has been shown in some previous studies to identify patients with Dukes' B disease who could benefit from adjuvant treatment. ${ }^{3}$ In CRC, patients within a given tumour stage display considerable prognostic heterogeneity, because they appear to be phenotypically and genotypically heterogeneous. Thus, it is generally believed that multiple genetic factors are involved in the pathogenesis of metastases, so that multiple tumour markers applied to the same tissue may be required. ${ }^{19}$ The sensitivity of a single mRNA

Table 2 Absolute expression of CEA, CK20, and GCC mRNA detected in Dukes' B and C colorectal cancer by R-PCR, and the presence (in bold) or absence of cancer specific expression based on the cutoff values defined in the text

\begin{tabular}{|c|c|c|c|c|c|c|c|}
\hline \multirow[b]{2}{*}{ Patient } & \multirow[b]{2}{*}{ Stage } & \multicolumn{2}{|l|}{ CEA } & \multicolumn{2}{|l|}{ CK20 } & \multicolumn{2}{|l|}{ GCC } \\
\hline & & FT & PET & FT & PET & FT & PET \\
\hline 1 & $B$ & $3.31 \times 10^{11}$ & $4.82 \times 10^{9}$ & $7.39 \times 10^{7}$ & $8.90 \times 10^{7}$ & - & - \\
\hline 2 & B & $8.03 \times 10^{11}$ & $6.07 \times 10^{9}$ & $2.56 \times 10^{7}$ & $1.70 \times 10^{6}$ & - & - \\
\hline 3 & B & NA & $1.25 \times 10^{9}$ & NA & - & NA & - \\
\hline 4 & B & NA & $8.27 \times 10^{9}$ & NA & $1.99 \times 10^{5}$ & NA & - \\
\hline 5 & $B$ & $2.93 \times 10^{11}$ & $1.83 \times 10^{9}$ & - & - & $3.32 \times 10^{8}$ & - \\
\hline 6 & B & $1.34 \times 10^{10}$ & $1.47 \times 10^{9}$ & - & - & $7.16 \times 10^{9}$ & - \\
\hline 7 & B & $2.34 \times 10^{13}$ & $1.72 \times 10^{10}$ & $2.77 \times 10^{8}$ & $5.97 \times 10^{7}$ & $1.39 \times 10^{11}$ & $8.64 \times 10^{9}$ \\
\hline 8 & B & $2.19 \times 10^{9}$ & $2.13 \times 10^{9}$ & - & - & - & $1.09 \times 10^{9}$ \\
\hline 9 & B & $5.59 \times 10^{9}$ & $2.17 \times 10^{8}$ & - & - & - & - \\
\hline 10 & $B$ & $1.88 \times 10^{9}$ & - & - & $2.10 \times 10^{7}$ & - & - \\
\hline 11 & B & $1.09 \times 10^{13}$ & $1.36 \times 10^{9}$ & $2.94 \times 10^{8}$ & - & - & $1.10 \times 10^{10}$ \\
\hline 12 & $\mathrm{C}$ & $3.54 \times 10^{12}$ & $1.57 \times 10^{12}$ & $6.28 \times 10^{8}$ & $5.48 \times 10^{7}$ & $4.35 \times 10^{11}$ & - \\
\hline 13 & C & $1.61 \times 10^{13}$ & $8.38 \times 10^{11}$ & $1.87 \times 10^{7}$ & $2.07 \times 10^{8}$ & $1.53 \times 10^{12}$ & $8.47 \times 10^{7}$ \\
\hline 14 & C & $9.88 \times 10^{13}$ & $1.26 \times 10^{9}$ & $2.43 \times 10^{7}$ & $7.53 \times 10^{7}$ & - & - \\
\hline 15 & C & $1.13 \times 10^{12}$ & $1.42 \times 10^{11}$ & $7.56 \times 10^{8}$ & $2.24 \times 10^{8}$ & - & - \\
\hline 16 & $\mathrm{C}$ & $1.20 \times 10^{15}$ & $6.30 \times 10^{10}$ & $2.95 \times 10^{7}$ & $2.42 \times 10^{7}$ & $7.51 \times 10^{12}$ & $1.88 \times 10^{7}$ \\
\hline 17 & $\mathrm{C}$ & NA & $3.60 \times 10^{10}$ & NA & $3.84 \times 10^{3}$ & NA & $2.92 \times 10^{10}$ \\
\hline 18 & C & $9.76 \times 10^{12}$ & $2.89 \times 10^{11}$ & $1.65 \times 10^{8}$ & $9.69 \times 10^{7}$ & $4.60 \times 10^{11}$ & $6.62 \times 10^{10}$ \\
\hline 19 & C & $2.14 \times 10^{13}$ & $8.14 \times 10^{10}$ & $4.14 \times 10^{9}$ & - & $7.90 \times 10^{11}$ & $1.07 \times 10^{9}$ \\
\hline 20 & C & NA & $2.64 \times 10^{9}$ & NA & $7.54 \times 10^{7}$ & NA & - \\
\hline 21 & C & $1.08 \times 10^{11}$ & $3.87 \times 10^{10}$ & $3.88 \times 10^{5}$ & $6.17 \times 10^{7}$ & $3.57 \times 10^{9}$ & $5.83 \times 10^{10}$ \\
\hline 22 & C & $1.65 \times 10^{11}$ & $1.36 \times 10^{12}$ & $7.36 \times 10^{8}$ & $1.30 \times 10^{10}$ & $2.27 \times 10^{9}$ & - \\
\hline 23 & C & $4.11 \times 10^{11}$ & $2.24 \times 10^{9}$ & $7.23 \times 10^{5}$ & - & $8.89 \times 10^{8}$ & - \\
\hline 24 & C & $2.76 \times 10^{12}$ & $5.86 \times 10^{11}$ & $2.01 \times 10^{2}$ & - & $8.34 \times 10^{9}$ & $1.93 \times 10^{9}$ \\
\hline 25 & C & $2.90 \times 10^{12}$ & $4.11 \times 10^{9}$ & $5.62 \times 10^{7}$ & $4.72 \times 10^{7}$ & $1.27 \times 10^{10}$ & - \\
\hline 26 & $C$ & $2.19 \times 10^{10}$ & $4.29 \times 10^{9}$ & $2.11 \times 10^{8}$ & - & $1.09 \times 10^{10}$ & - \\
\hline
\end{tabular}


marker in the detection of metastases by RT-PCR may be inadequate because of tumour heterogeneity in gene expression. ${ }^{20}{ }^{21}$ Thus, we took advantage of the fact that CEA, CK20, and GCC are expressed constitutively in normal colon mucosa and most CRCs, but show relatively low expression in lymphatic tissue, ${ }^{22}$ to develop a R-PCR based molecular assessment of tumour stage using all three biomarkers.

Because traditional RT-PCR technology is at best semiquantitative, it has been difficult to differentiate between baseline gene expression in normal tissues and the increased gene expression associated with cancer, raising the concern of false positive results. ${ }^{23-25}$ "Normal" baseline expression has been reported in the analysis of $\mathrm{CEA}^{24}$ and CK20. ${ }^{26}$ This is the rationale behind the determination of the threshold values of constitutive expression of these markers, or the development of quantitative techniques to distinguish between tumour specific gene expression and low level background transcription of such markers. ${ }^{27}$ The phenomenon of illegitimate or ectopic transcription of CEA and CK20 mRNA in our Dukes' A LNs at low, constitutive values supports the difficulty of interpreting the expression profiles determined by conventional RT-PCR, and demonstrates the potential new information that quantitative R-PCR analysis can provide.

Several studies have shown that the gel electrophoresis based RT-PCR assay has a high sensitivity for detecting disseminated micrometastases of CRC to regional LNs. Waldman and colleagues ${ }^{28}$ reported that GCC mRNA was detected in LNs from one third of patients with Dukes' B CRC and from all patients with Dukes' C CRC. Similarly, in a study by Cagir et al, ${ }^{29}$ GCC was detected in the LNs of 10 patients with stage II disease who developed recurrent disease up to three years after diagnosis. Our data confirm the ability of GCC specific PCR to detect the presence of micrometastases. In addition, our quantitative R-PCR assay focuses on the quantification of disseminated cancer cells in dissected LNs of CRC to provide a more sensitive and reliable measurement. The potential of quantitative PCR and the improvement it provided compared with conventional RT-PCR for the detection of occult lymph node metastases has been demonstrated in oesophageal cancer ${ }^{30}$ and during intraoperative assessment of sentinel lymph nodes. ${ }^{31}$

\begin{abstract}
"Biomarkers that have been accepted as universal metastatic markers for a given tumour are expressed differently, and this may be related to the clonal selection that takes place in the primary carcinoma when cells are entering the metastatic cascade"
\end{abstract}

The main conclusion of our study is that different metastatic biomarkers can give different results when applied to the same material, and that the same biomarker can give different results when applied to differently processed tissues. From a biological viewpoint, this observation indicates that biomarkers that have been accepted as universal "metastatic markers" for a given tumour are expressed differently, and this may be related to the clonal selection that takes place in the primary carcinoma when cells are entering the "metastatic cascade". In other words, markers that are faithfully represented in the original tumour may not be expressed by the small number of malignant cells that initiate the micrometastasis process. More importantly, this biological variability has a clear consequence for those assays that aim to use the detection of micrometastases in the clinical setting, because it is clear now that a "metastatic biomarker" may not be accurate just because it is detectable in the primary neoplasm. Indeed, our results support those who advocate a multimarker approach to micrometastases $^{1031}$ or minimal residual disease detection, ${ }^{11}$ and lend further credence to the idea that no universal metastatic marker can be presumed for CRC.

The discrepancy of results between PET and FT is another important clinical observation. Our data suggest that it cannot be presumed that formalin fixed PET is a reliable support for quantitative PCR detection of small numbers of metastatic malignant cells (table 1). We showed that the quality of the mRNA extracted from PET could alter the efficacy of any tumour marker in the detection of micrometastases. As would be expected, mRNA preservation was better in the frozen material, and we detected the presence of CEA, CK20, and GCC in a larger number of frozen LNs than fixed LNs. Our results show that measures to enhance RNA preservation in archival tissues must be carefully considered when developing a sensitive, specific, and reliable R-PCR detection assay.

We also found that some LNs with morphological evidence of metastasis (Dukes' C) did not show expression of certain biomarkers. In the FTs, this could perhaps be explained by the fact that metastases were only present in the half of the LN that was fixed and paraffin wax embedded for routine diagnosis and were absent from the FT sample. Incidentally, all the Dukes' $\mathrm{C}$ cases were thoroughly reviewed by two pathologists and the tumour was found in all the paraffin wax blocks labelled as positive LN metastases. The fact that the PCR amplification of the housekeeping internal control gene (with a base pair size similar to the PCR product of the biomarkers) and the positive cell line control worked well indicates that this discrepancy cannot be attributed to technical reasons.

One patient with Dukes' B disease (patient 7, table 2), in whom all three metastatic biomarkers were present in both tissue types, suffered a relapse and died within two years of follow up. However, our patients were all too recently diagnosed to allow a reliable correlation of the longterm survival rate among the prognostically heterogeneous patients with Dukes' B disease. The accumulation of data for a larger sample size and a longer follow up period is planned to enhance the clinical value of our study.

In summary, our study highlights the complexity of gene expression in microscopically occult LN metastases. It appears that, with the current state of the art technology, the discrepancy between the biomarkers most frequently used in the literature is too great to validate the clinical relevance of any single marker assay in the routine diagnostic setting for this purpose. The search for more promising,

\section{Take home messages}

- We assessed the nodal expression of the CEA (carcinoembryonic antigen), CK20 (cytokeratin 20), and GCC (guanylyl cyclase C) genes in patients with colorectal cancer (CRC) and found considerable discordance in the positive detection of the three putative tumour markers

- We also found variability in results using either frozen or paraffin wax embedded tissues

- This suggests that using a single tumour marker and one tissue type (either frozen or paraffin wax embedded) may not be adequate to detect micrometastasis, and challenges the concept of universal markers for molecular CRC metastatic detection

- Multiple tumour markers would predict more accurately the metastatic potential of Dukes' B CRCs 
cancer specific biomarkers (for example, gene hypermethylation status $)^{32}$ is needed to establish further the clinical usefulness of the detection of microscopically occult nodal micrometases by molecular methods.

\section{ACKNOWLEDGEMENTS}

We thank Ms S Liang of the Clinical Trials and Epidemiology Research Unit, Singapore for her contribution to the statistical analysis of our data. This work was supported by grants from the National Medical Research Council, Singapore (NMRC-R-179-000013-214 and NMRC/0383/1999) and the Singapore Cancer Society (SCS-R-179-000-014-593) to MST and ESCK.

\section{Authors' affiliations}

S L Kong, E S C Koay, Department of Pathology, National University of Singapore, 10 Kent Ridge Crescent, Singapore 119260

M Salto-Tellez, Molecular Diagnosis Centre, Department of Laboratory Medicine, National University Hospital, Singapore 119074

A P K Leong, Department of Surgery, National University Hospital, Singapore

Y H Chan, Clinical Trials and Epidemiology Research Unit, 226 Outram Road, Singapore 169039

\section{REFERENCES}

1 Toribara NW, Sleisenger MH. Screening for colorectal cancer. N Engl J Med 1995:332:861-7.

2 Jessup JM, McGinnis LS, Steele GDJ, et al. The National Cancer Data Base: report on colon cancer. Cancer 1996;78:918-26.

3 Lindblom A. Improved tumor staging in colorectal cancer [editorial]. N Engl J Med 1998;339:264-5.

4 Liefers GJ, Cleton-Jansen AM, van de Velde CJ, et al. Micrometastases and survival in stage II colorectal cancer. N Engl J Med 1998;339:223-8.

5 Lassmann S, Baver M, Soong R, et al. Quantification of CK20 gene and protein expression in colorectal cancer by RT-PCR and immunohistochemistry reveals inter- and intratumour heterogeneity. J Pathol 2002;198:198-206.

6 Gardner B, Feldman J. Are positive axillary nodes in breast cancer markers for incurable disease [discussion]? Ann Surg 1993;218:270-5.

7 Salto-Tellez M, Kong SL, Leong APK, et al. Intrinsic variability in the detection of micrometastases in lymph nodes for re-staging of colorectal cancer: effect of individual markers and tissue sample. Eur J Cancer 2003;39:1234-41.

8 Martell M, Gomez J, Esteban Jl, et al. High-throughput real-time reverse transcription-PCR quantitation of hepatitis C virus RNA. J Clin Microbiol 1999;37:327-32.

9 Nakao M, Janssen JW, Flohr T, et al. Rapid and reliable quantification of minimal residual disease in acute lymphoblastic leukemia using rearranged immunoglobulin and T-cell receptor loci by LightCycler technology. Cancer Res 2000;60:3281-9.

10 Mitas M, Mikhitarian K, Walters C, et al. Quantitative real-time RT-PCR detection of breast cancer micrometastasis using a multigene marker panel. Int J Cancer 2001:93:162-71.

11 Northover J. The use of prognostic markers in surgery for colorectal cancer. Eur J Cancer 1995;31A:1207-9.

12 Moll R, Zimbelmann R, Goldschmidt MD, et al. The human gene encoding cytokeratin 20 and its expression during fetal development and in gastrointestinal carcinomas. Differentiation 1993;53:75-93.
13 Gyles CL. Escherichia coli cytotoxins and enterotoxins. Can J Microbiol 1992;38:734-46.

14 Carrithers SL, Parkinson SJ, Goldstein S, et al. Escherichia coli heat-stable toxin receptors in human colonic tumors. Gastroenterology 1994; 107:1653-61.

15 Evans $\mathrm{H}$, Sillibourne J. Detection of the $\mathrm{Bcr}-\mathrm{Ab} /$ gene in $\mathrm{CML} / \mathrm{ALL}$ patients. Promega Notes Magazine 1996:57:21-3.

16 Gerhard M, Juhl H, Kalthoff $\mathrm{H}$, et al. Specific detection of carcinoembryonic antigen-expressing tumor cells in bone marrow aspirates by polymerase chain reaction. J Clin Oncol 1994;12:725-9.

17 Funaki NO, Tanaka J, Ohshio G, et al. Cytokeratin 20 mRNA in peripheral venous blood of colorectal carcinoma patients. $\mathrm{Br} J$ Cancer 1998;77:1327-32.

18 Carrithers SL, Barber MT, Biswas S, et al. Guanylyl cyclase C is a selective marker for metastatic colorectal tumors in human extraintestinal tissues. Proc Natl Acad Sci U S A 1996;93:14827-32.

19 Guan RJ, Van Dam J. Molecular detection of micrometastases: science on stage [review]. Am J Gastroenterol 1999;94:3062-4

20 Mori $M$, Mimori K, Inove $H$, et al. Detection of cancer micrometastases in lymph nodes by reverse transcriptase-polymerase chain reaction. Cancer Res 1995:55:3417-20.

21 Noguchi S, Aihara T, Motomura K, et al. Detection of breast cancer micrometastases in axillary lymph nodes by means of reverse-transcriptase polymerase chain reaction. Am J Pathol 1996;148:649-56.

22 Futumura M, Takagi Y, Koumura H, et al. Spread of colorectal cancer micrometastases in regional lymph nodes by reverse transcriptase-polymerase chain reactions for carcinoembryonic antigen and cytokeratin 20. J Surg Oncol 1998;68:34-40.

23 Datta YH, Adams PT, Drobyski WR, et al. Sensitive detection of occult breast cancer by the reverse-transcriptase polymerase chain reaction. J Clin Oncol 1994; 12:475-82.

24 Bostick PJ, Chatterjee S, Chi DD, et al. Limitations of specific reversetranscriptase polymerase chain reaction markers in the detection of metastases in the lymph nodes and blood of breast cancer patients. J Clin Oncol $1998 ; 16: 2632-40$

25 Merrie AE, Yun K, Gunn J, et al. Analysis of potential markers for detection of submicroscopic lymph node metastases in breast cancer. $\mathrm{Br} J$ Cancer 1999;80:2019-24.

26 Jung R, Petersen K, Krüger W, et al. Detection of micrometastasis by cytokeratin 20 RT-PCR is limited due to stable background transcription in granulocytes. Br J Cancer 1999;81:870-3.

27 Champelovier P, Mongelard F, Seigneurin D. CK20 gene expression: technical limits for the detection of circulating tumor cells. Anticancer Res 1999:19:2073-8.

28 Waldman SA, Cagir B, Rakinic J, et al. Use of guanylyl cyclase C for detecting micrometastases in lymph nodes of patients with colon cancer. Dis Colon Rectum 1998:41:310-15.

29 Cagir B, Gelmann A, Park J, et al. Guanylyl cyclase C messenger RNA is a biomarker for recurrent stage II colorectal cancer. Ann Intern Med 1999;131:805-12.

30 Godfrey TE, Raja S, Finkelstein SD, et al. Prognostic value of quantitative reverse-transcription-polymerase chain reaction in lymph node-negative esophageal cancer patients. Clin Cancer Res 2001;7:4041-8.

31 Raja S, El-Hefnawy T, Kelly LA, et al. Temperature-controlled primer limit for multiplexing of rapid, quantitative reverse transcription-PCR assays: application to intraoperative cancer diagnostics. Clin Chem 2002;48:1329-37

32 Evron E, Dooley WC, Umbricht CB, et al. Detection of breast cancer cells in ductal lavage fluid by methylation-specific PCR. Lancet $2001 ; 357: 1335-6$ 